\title{
Determining the Causes of Scanning Distortions in SEM and FIB
}

\author{
Mariusz Pluska ${ }^{1}$, Andrzej Czerwinski ${ }^{1}$, Marek Wzorek ${ }^{1}$, Marcin Juchniewicz ${ }^{1}$, Jerzy Kątcki ${ }^{1}$ \\ ${ }^{1 .}$ Institute of Electron Technology, Al. Lotnikow 32/46, Warsaw 02-668, Poland
}

Scanning distortions are a well-known issue in the scanning electron microscopy (SEM) [1, 2], and the problem is related to the focused ion beam (FIB) instrumentation as well [3]. The characteristic jagging of vertical edges in SEM images may disturb observations and impede imaging. The same effect occurring during Focused Ion Beam (FIB) operations causes imperfect patterning of designed shapes and affects the FIB micro/nano machining. In effect, it physically harms or damages patterned structures or devices.

The image distortions are attributed most frequently to the presence of alternating magnetic field [4] (or broadly speaking to electromagnetic interference - EMI). Their acoustic origin has been also considered [5]. Accurate determining the real causes of observed imaging and patterning distortions is necessary for their removal. The presented studies showed that although both influences are probable, there is a way to distinguish which one (EMI or acoustic) is the dominant cause of distortions. Such knowledge can prove to be useful. The most popular solutions to the distortion problem are the magnetic field cancellation systems, which installation may be however expensive and uncomfortable. Moreover, it may not help, what makes matters worse. Therefore, it is recommended to check with the use of the proposed methods, whether the main problem is not caused e.g. by a noisy air conditioner or an equipment cooling fan.

The influences of EMI and the acoustic noise are distinguishable because they act on the electron/ion beam in different ways. The method to separate them is based on the measurement of the level of the image distortion for several working distances. The peak-to-peak value of edge deformation is a good measure for the level of distortion (inset in Fig. 1a).

EMI acts on the electron or ion beam directly through the Lorentz force The impact of the external electromagnetic field is usually reduced by the SEM/FIB chassis. However, especially for low frequencies (e.g. 50-60 Hz), it is impossible to remove EMI impact completely [6]. The effect of distorting electromagnetic field accumulates along the entire path of a charged particle. Therefore, the longer working distance we use, the stronger distortions we observe. The path of the electron or ion under the influence of the uniformly distributed electromagnetic field is a quadratic curve similar to the trajectory in the gravitational field. If the peak-to-peak values of the deformation of a specimen edge are measured for a number of working distances and the plotted points fit the quadratic function, then it may be concluded that the distortions are caused by the electromagnetic field (Fig. 1a). The plot shown in figure 1a was registered in SEM for electron energy of $5 \mathrm{keV}$ and intentionally generated distortions: magnetic field $B=220 \mathrm{nT}$, frequency $f=50 \mathrm{~Hz}$.

The acoustic noise acts on the mechanical components of the instrument. The acoustic wave propagates within the chassis of the instrument and if it matches the resonance frequency of any component (e.g. a piezo-driven specimen stage), then the vibration occurs and gains in intensity. Therefore, the frequency response of the SEM or FIB instrument to the acoustic distortions consists of several narrow peaks (inset in Fig 1b). The present investigation showed that the ambient noise in the instrument environment may contain resonance frequencies of its particular components and therefore it can evoke strong vibrations 
visible in SEM/FIB images. It was found that for the distortions caused by the acoustic noise there is no predictable dependence between the working distance and the level of the image deformation (Fig. 1b). The measurement of the peak-to-peak value of specimen edge deformation versus working distance in case when it is caused by the noise, does not match the quadratic function. The plot shown in figure 1a was registered in SEM for electron energy of $5 \mathrm{keV}$ and intentionally generated acoustic distortions: SPL (sound pressure level) $=75 \mathrm{~dB}, f=216 \mathrm{~Hz}$ (which is one of the resonance frequencies).

In conclusion, the presented study describes a simple and useful method for determining if the observed distortions are caused by EMI or by acoustic noise. This is an important guideline when considering methods aimed at SEM/FIB images improvement and FIB micro/nano machining refinement [7].

[1] D.H. Kim, S.J. Kim and S.K. Oh, Nuclear Instruments and Methods in Physics Research A 620.2(2010), p. 112.

[2] M. Pluska et al, Journal of Microscopy 224.1 (2006), p. 89.

[3] M. Pluska et al, Nuclear Instruments and Methods in Physics Research B (2014)

doi:10.1016/j.nimb.2014.11.020.

[4] A.E. Vladar, "Scanning electron microscopy in real world environment" (2003), www.nanobuildings.com.

[5] K.O. Jung, S.J. Kim and D.H. Kim, Nuclear Instruments and Methods in Physics Research A 676 (2012), p. 5.

[6] C.S. Cheung, "Shielding Effectiveness of Superalloy, Aluminum, and Mumetal Shielding Tapes." (2009), Master's Theses and Project Reports, p. 126.

[7] The research was partially supported by the grant LIDER/26/169/L-3/11/NCBR/2012 of National Centre for Research and Development, Poland.

a)

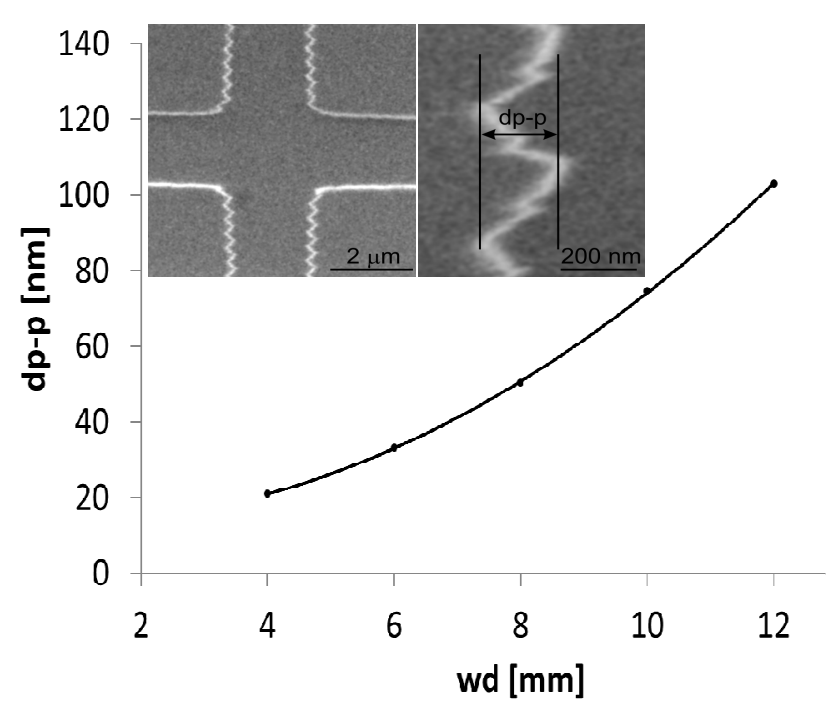

b)

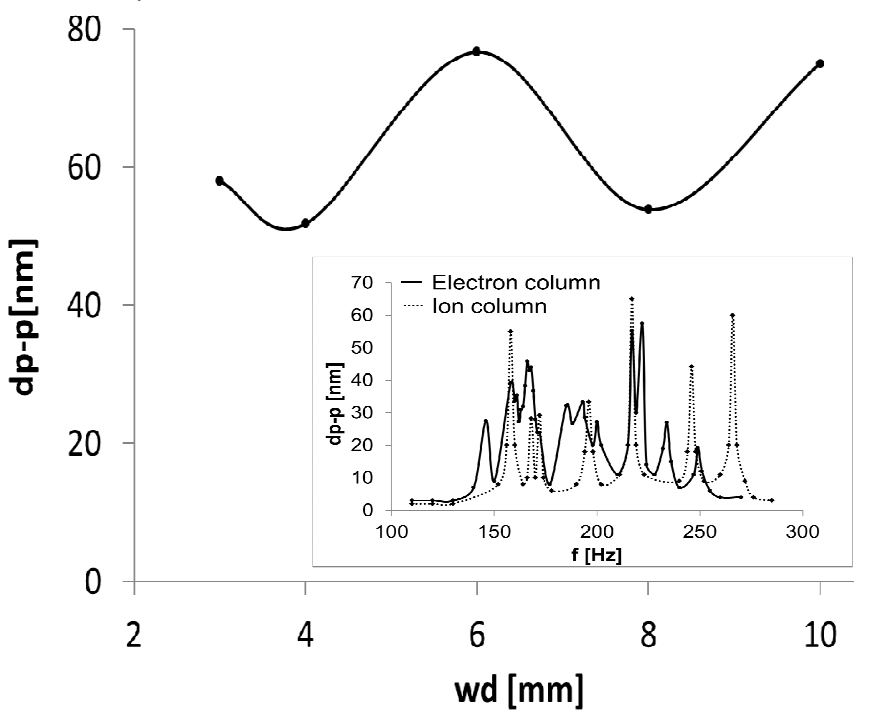

Figure 1. Plots of the peak-to-peak value $d_{p-p}$ of the edge deformation versus the working distance (wd) for intentionally generated magnetic (a) and acoustic (b) distortions in SEM. Inset in fig. 1a shows the method to measure the distortion level. Inset in fig. 1b shows measured frequency response of exemplary instrument (Helios NanoLab 600 Dual Beam FIB) to the acoustic vibration. 